\title{
Re-Model the Relation of Vector Indices, Meteorological Factors and Dengue Fever
}

\section{Yao-Ting Tseng ${ }^{1}$, Fong-Shue Chang ${ }^{2}$, Day-Yu Chao ${ }^{2^{*}}$ and le-Bin Lian ${ }^{3^{*}}$}

${ }^{1}$ Department of Mathematics, National Changhua University of Education, Changhua, 50007, Taiwan

${ }^{2}$ Graduate Institute of Microbiology and Public Health, Nation Chung Hsing University, Taichung 40227, Taiwan

${ }^{3}$ Graduate Institute of Statistics and Information Science, National Changhua University of Education, Changhua, 50007, Taiwan

\section{Abstract}

Background: Dengue is the most rapidly expanding and spreading mosquito-borne viral disease in tropical and subtropical countries. In Taiwan, dengue incidence clustered in Southern part, especially Kaohsiung in the past decade.

Aim: The spatial and temporal patterns of dengue transmission in Taiwan from 2005 to 2012 were examined to investigate the occurrence of dengue fever (DF) patients and its association with immature and adult mosquito indices, and its interaction with meteorological factors and household density.

Methods: Three databases were spatially and temporally linked, including the comprehensive chart records of DF cases and vector surveillance data in Kaohsiung, as well as the meteorological and environmental information from 2005 to 2012. A case-crossover study design was used to explore the effects of mosquito indices and weather on the risks of DF, and conditional logistic regression was applied to estimate the odds ratios (OR).

Results: Results showed immature mosquito indices had significant positive association with DF in the medium and high household density areas (e.g., adjusted ORs of Breteau index were 1.04, 95\% Cl=[1.02, 1.06] and 1.06, $\mathrm{Cl}=[1.04$, 1.08] respectively), while adult mosquito index was significant to all low/med/high household densities (adjusted ORs of Aedes aegypti index were 1.29, $\mathrm{Cl}=[1.23,1.36] ; 1.49, \mathrm{Cl}=[1.37,1.61]$ and $1.3, \mathrm{Cl}=[1.21,1.39]$ respectively). Meanwhile, combination with 2-week lag rainfall, 2-month lag rainfall, 2-week lag temperature and relative humidity, resulted better prediction of DF incidence.

Conclusion: Meteorological conditions affect DF occurrence in a nonlinear way, and a single time-point rainfall variable is insufficient to fit it. Our study suggested that short-lag (last 2 weeks) conditions of moderate rainfall, moderate temperature and high humidity, in combination with a long-lag heavy rainfall were related to higher probability of DF incidence. $\mathrm{BI}$ and $\mathrm{Cl}$ are useful predictors for DF occurrence in medium and high household density areas, but not in the low density areas.

Keywords: Case-crossover study; Conditional logistic regression; Household density; Vector surveillance; Aedes aegypti; Meteorology

\section{Introduction}

Dengue is the most rapidly expanding and spreading mosquitoborne viral disease in tropical and subtropical countries [1]. This disease is mainly transmitted by Aedes aegypti and Aedes albopictus [2]. The incidence of dengue has increased by 30 -fold in the past 50 years [1]. Approximately 2.5 billion people in more than 100 countries are currently under the risk of dengue viral infection, with the majority living in the Asia-Pacific region [3]. Dengue virus has four serotypes (DENV-1-DENV-4), resulting in a wide spectrum of clinical manifestations, including dengue fever (DF), dengue hemorrhagic fever, and dengue shock syndrome. No vaccine or anti-viral drug is currently available for dengue patients.

Transmission of DENV is maintained by horizontal transfer in an A. aegypti-human cycle, although vertical transmission has also been reported [4]. Mosquito infection begins when females imbibe viremic blood from a human host and survive an extrinsic incubation period of 7-14 days [5,6]. A few commonly used Stegomyia indices are as follows: the premise or house index (HI: percentage of houses infested with larvae and/or pupae), container index (CI: percentage of waterholding containers infested with larvae and/or pupae), and Breteau index (BI: number of positive containers per 100 houses) or oviposition trap (ovitrap) data. All these indices are intended to detect the presence or absence of $A$. aegypti rather than the relative abundance of adult virus transmitting mosquitoes $[7,8]$. Adult $A$. aegypti index (AI) can be determined by collecting mosquitoes with backpack aspirators or sweep nets [9]. Although adult population density has been linked to the epidemiologically important dengue incident rate, the implementation is labor intensive and usually expensive under limited budget $[10,11]$.

Numerous efforts have been made to investigate the relationship between these mosquito indices and DENV transmission. However, several researchers repeatedly reported weak association and that DENV transmission frequently occurs even when A. aegypti population densities are low [8,12-16]. Different factors influence DENV transmission, including temporal and meteorological effects on mosquito life stages, larval mortality, heterogeneity in container productivity, variation in susceptibility of the human population to DENV infection, and spatial heterogeneities in vector density $[8,11,15,17,18]$. Failure to

*Corresponding author: le-Bin Lian, Graduate Institute of Statistics and Information Science, National Changhua University of Education, Changhua, 50007, Taiwan, Tel: 886-4-7232105 ext 3201; Fax: 886-4-7211192; E-mail: maiblian@cc.ncue.edu.tw

Day-Yu Chao, Graduate Institute of Microbiology and Public Health, Nation Chung Hsing University, Taichung 40227, Taiwan, Tel: 886-4-22840694; Fax: 886-422852186; E-mail: dychao@nchu.edu.tw

Received December 20, 2015; Accepted January 12, 2016; Published January 19, 2016

Citation: Tseng YT, Chang FS, Chao DY, Lian IB (2016) Re-Model the Relation of Vector Indices, Meteorological Factors and Dengue Fever. J Trop Dis 4: 200. doi:10.4172/2329-891X.1000200

Copyright: ( 2016 Tseng YT, et al. This is an open-access article distributed unde the terms of the Creative Commons Attribution License, which permits unrestricted use, distribution, and reproduction in any medium, provided the original author and source are credited. 
consider these factors may lower the prediction accuracy for DENV. Furthermore, administrative inconsistency among different spatial units may also cause geographical differences of the vector and dengue case data, which can mask potential relationship [19]. An ideal study design that considers the spatial and temporal aspects of these variables should provide better understanding on the relationship between $A$. aegypti population densities, DENV transmission, and disease incidence.

Kaohsiung City, a modern metropolis of 1.5 million people, has been affected by different serotypes of DENV and becomes the focus of DENV activity in Taiwan over the last decades [20-23]. During 20022011, Kaohsiung City had annual outbreaks of variable scales, resulting in more than 6,000 confirmed cases [24]. Since 2005, the Department of Health of Kaohsiung City Government has been initiating surveillance activities by using specially trained personnel. A previous study suggested that AI from 2005 to 2009 shows temporal correlation with the peak of the DF activity, with 1-2-month lag period [25]. However, the association between different vector indices and the occurrence of dengue cases has been not completely evaluated. In the present study, the case-crossover study design was applied to 8-year longitudinal data focusing on Kaohsiung City in Taiwan, where the most dengue incidences occurred. This study investigated the occurrence of dengue patients and its association with (1) different immature mosquito indices, (2) adult mosquito density, and (3) their interplay with meteorological factors and household density.

\section{Aim}

This study primarily depicted the association between the occurrence of DF and the vector surveillance factors including $\mathrm{BI}, \mathrm{AI}$, $\mathrm{CI}$, and $\mathrm{HI}$; the meteorological factors including rainfall, temperature, and relative humidity; and a few resident conditions including household density.

\section{Materials and Methods}

\section{Study area}

The government combined Kaohsiung City with Kaohsiung County into one unified administrative unit after December 25, 2010. Our study area includes the former Kaohsiung City and adjacent districts from the former Kaohsiung County, including Fongshan, Daliao, and Linyuan (Figure 1). The area is located from $120^{\circ} 10^{\prime} 32^{\prime \prime}$ to $121^{\circ} 01^{\prime} 15^{\prime \prime}$ east longitudes and $22^{\circ} 28^{\prime}$ to $23^{\circ} 28^{\prime}$ north latitudes. Kaohsiung City is a standard subtropical region, with annual average rainfall from 1796.7-2821.4 mm concentrated from May to September. In addition, the annual average temperature is from $24.9^{\circ} \mathrm{C}-25.7^{\circ} \mathrm{C}$, with the lowest average of $11.6^{\circ} \mathrm{C}$ in February and the highest average of $31.5^{\circ} \mathrm{C}$ in June.

\section{Data sources}

We used the following four databases to collect data from 2005 to 2012: (1) dengue patient surveillance, (2) vector surveillance, (3) household registration, and (4) meteorological data. The first three databases were obtained from the Department of Health, Kaohsiung City Government, and the last database was archived from the databank of Environmental Protection Administration (EPA), Taiwan. The detailed description of each surveillance system can be referred to the EPA [26]. Noting that DF is classified as a legal communicable diseases in Taiwan, which means that all the cases have to be reported to the government. The county/city government is responsible for ascertaining each cases and reporting the record to Taiwan Center of Disease Control, as well as all the necessary measures for DF

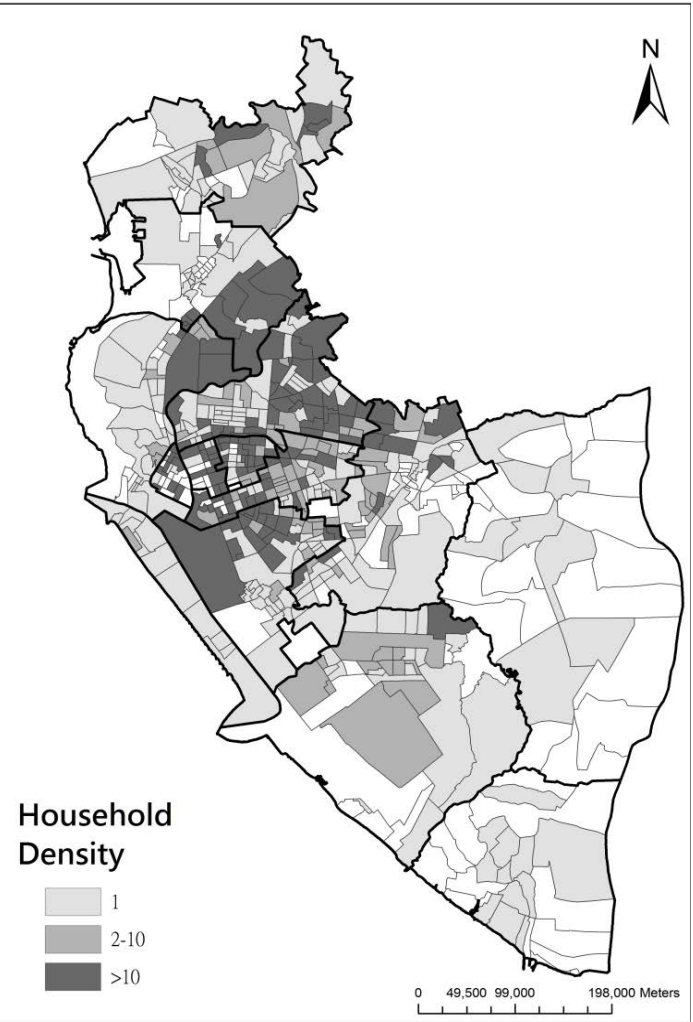

Figure 1: Geographic distribution of household density in Kaohsiung

prevention, including vector surveillance. Therefore the Department of Health of Kaohsiung has the most accurate DF surveillance data. Notably, considering the administrative change mentioned previously, the former Kaohsiung City has 8-year surveillance records, whereas the records of the districts from former Kaohsiung County started from late 2010. All the ascertained DF cases were collected via 3 types of surveillance: passive, active and semi-active. Most of the cases were mainly from passive and semi-active surveillance. In passive surveillance, dengue-like illness was reported by health care workers, and confirmed by local health authorities, while in semi-active surveillance, fever cases are investigated in residential areas, schools, and work places with epidemiological linkage, and specimens are taken once confirmed dengue cases are identified [27].

The records include the date of ascertainment, residency, age at the time of diagnosis, and gender. Notably, "Li" is the smallest administrative unit in Taiwan, and more than 900 Lis are present within the study area. In this study, the address of a patient is defined as Li. DF case is an acute febrile viral disease and fever $\left(>38^{\circ} \mathrm{C}\right)$, with at least two clinical symptoms, such as intense headache, nausea, fatigue, retroorbital pain, myalgia, arthralgia, and skin rash [28].

Second, vector surveillance data including AI, BI, CI, and $\mathrm{HI}$ were summarized as the averaged measurement of 2-week period for each Li. However, we excluded the Aedes albopictus that activities at outdoor, because the Kaohsiung is an urban city [2]. The frequency of vector surveillance in Kaohsiung depends on the former DF incidence rate over the past years in each area. By the government definition, these Lis were considered to be of high incidence and surveyed once every week. The middle incidence and low incidence were surveyed in 
monthly and tri-monthly basis, respectively.

To explore the effect of building style on DENV transmission, we divided all the Lis into three groups according to its household density levels. Notably, each doorplate of a building has its coordinates located by satellite and is shown in the geographical information system map. For each doorplate, we sum up the number of households with similar coordinates and classified them as "1 household," "2-10 households," and "> $>10$ households." For each $\mathrm{Li}$, if the "1 household" building comprises more than $50 \%$ of all the buildings, then, the $\mathrm{Li}$ is regarded as low household density. For others, if a Li has higher proportion of " $2-10$ households" buildings than "> 10 households," then, the $\mathrm{Li}$ is regarded as medium household density; otherwise, the $\mathrm{Li}$ is classified as high household density.

Nine EPA monitor stations scattered in Kaohsiung collected the meteorological data. These data provide information, such as daily accumulative rainfall, daily mean relative humidity, and daily mean temperature. For each $\mathrm{Li}$, its meteorological datum was calculated by using inverse distance weighting interpolation. All meteorological data were trisected into three levels (low, medium, and high), roughly according to the 33rd and 66th percentiles. As a result, the corresponding low, medium, and high accumulated rainfall of 8-14 days before diagnosis (i.e., 1-2 week lag) were 0-2.5 mm, 2.5-30.6 $\mathrm{mm}$, and $>30.6 \mathrm{~mm}$, respectively. Moreover, the accumulative rainfall of 29-56 days before diagnosis (5-8 week lag) were 0-56.2 mm, 56.2$197 \mathrm{~mm}$, and $>197 \mathrm{~mm}$. The $1-2$ week lag average temperatures of the tripartitions were $16^{\circ} \mathrm{C}-27.4{ }^{\circ} \mathrm{C}$ (low), $27.4{ }^{\circ} \mathrm{C}-29^{\circ} \mathrm{C}$ (medium), and $>29^{\circ} \mathrm{C}$ (high). The $1-2$ week lag average relative humidity percentages of the tripartitions were $52 \%-72.9 \%, 72.9 \%-77 \%$ and $>77 \%$.

\section{Statistics analysis}

A time-stratified case-crossover design [29-30] was used in this study. This method is used to investigate the effect of short-term exposure to risks that continuously change, occur, and measure. By using the self-controlled crossover design, cases act as their own control in periods when they are unexposed. Therefore, this method accounts for unmeasured confounding variables when these cases are constant over time within individuals. By using the same case in different (but insignificant) periods of time as its controls, the long-term effects, such as gender, social economic status, body mass index, habits, and household type, can be completely controlled.

Each patient presenting with DF for a specific condition was considered as a case on the date of diagnosis and as three possible controls on selected days, roughly 3,6 , and 9 weeks before the date of DF diagnosis. We defined "Week 1" as the first 7 days before the diagnosis, "Week 2" as the 8th-14th days before the diagnosis, and so on. For a case, the correspondent values of vector-borne (AI, BI, CI, and $\mathrm{HI}$ ) were based on the surveillance measurement within the 2-week period before the diagnosis (i.e., Week 1 and 2). Similarly, the period of measurements for the first control was set to be 3 weeks ahead of the case and that of the second control was set to be 6 weeks ahead of the case, and vice versa.

As for the meteorological factors, we considered the following variables:

- 2-week lag rainfall: for the case, this factor is defined as the average daily rainfall at the $\mathrm{Li}$ of the patient within the 2-week period prior to DF diagnosis (Week 1 to Week 2); and for the controls, this factor is the analogous average, but 3,6 , and 9 weeks earlier.
- 2-month lag rainfall: the average daily rainfall from Week 5 to Week 8 (2 months prior to DF diagnosis).

- 2-week lag temperature: the average daily temperature from Week 1 to Week 2.

- 2-week lag relative humidity: the average daily relative humidity from Week 1 to Week 2.

Considering that a DF case and its matched controls are from the same patient, their long-term factors, such as living environment, habits, and chronic health conditions, are sufficiently controlled. The effects of vector-borne and meteorological factors were compared between case and control days by the following model:

$$
\begin{aligned}
\log \text { it }(\mathrm{Y}) & =\beta_{0}+\beta_{1} \cdot V I+\beta_{2} \cdot R F_{11}+\beta_{3} \cdot R F_{12}+\beta_{4} \cdot R F_{21}+\beta_{5} \cdot R F_{22} \\
& +\beta_{6} \cdot \operatorname{Temp}_{1}+\beta_{7} \cdot \operatorname{Temp}_{2}+\beta_{8} \cdot R H_{1}+\beta_{9} \cdot R H_{2}
\end{aligned}
$$

where $\mathrm{Y}=1$ for case, and $=0$ for control, and VI is one of the vectorborne indices ( $\mathrm{AI}, \mathrm{BI}, \mathrm{CI}$, or $\mathrm{HI}$ ). $\mathrm{RF}_{11}, \mathrm{Temp}_{1}$, and $\mathrm{RH}_{1}$ are indicator variables, with a value 1 if the 2 -week lag rainfall, temperature, and relative humidity are between the (overall) $33 \mathrm{rd}$ and 66 th percentiles; otherwise, 0 . Similarly, $\mathrm{RF}_{12}, \mathrm{Temp}_{2}$, and $\mathrm{RH}_{2}$ have a value of 1 if the 2-week lag rainfall, temperature, and relative humidity are above the 66th percentiles. Furthermore, $\mathrm{RF}_{21}$ is an indicator variable, with value 1 if the 2-month lag rainfall is between the (overall) 33rd and 66th; otherwise, 0 . Similarly, $\mathrm{RF}_{22}$ has a value of 1 if the 2 -month lag rainfall is above the 66th percentiles.

Each stratum should ideally contain one case and three controls. However, the city government may not measure the indices every week in every Li. If any case does not have the vector measure in the correspondent Li in neither Week 1 nor Week 2 , or if none of the controls have the measure during the correspondent period, then the stratum is deleted. By contrast, if more than one measurement is conducted within a week, then the average is selected. May to December is the DF prevalent season, with DF cases of $98.7 \%$ among all cases of a year, and surveillance data are more complete in this period. Thus, we only considered the cases that occurred between May and December. After the exclusion, 2453 out of the 4570 DF cases (strata) (54\%) were used in the following analysis. We then estimated the association between vector-borne and the risk of DF using conditional logistic regression models, which was adjusted for the meteorological factors.

Each model contains only one index and adjusted by the meteorological factors to avoid the high co-linearity among the mosquito indices, namely, AI, BI, CI, and HI. All analyses were performed using the statistics software SAS 9.3. The study was approved by the Institutional Review Board IRB-R-05-002 of Taichung Hospital, Ministry of Health and Welfare.

\section{Results}

A total of 4570 indigenous dengue cases occurred during the period from 2005 to 2012 in Kaohsiung City, Southern Taiwan. Nearly 96\% of the cases occurred from August 1 to December 31. Given the design of our study, only the cases with chronically and spatially matched vector indices data were recruited. As a result, only 2453 cases or $54 \%$ among all cases were analyzed, with mean ages of $46.6,45.8$, and 44.5 in low, medium, and high household density areas, respectively. The gender distribution of DF patients was composed of $46.9 \%, 51.2 \%$, and $47.3 \%$ of males in low, medium, and high household density areas, respectively. Chi-square test was carried out, and no statistically significant differences were observed among all 4570 cases and the recruited 2453 
dengue cases in terms of residential area (number of $\mathrm{Li}$ ), gender, and age (Table 1). However, slightly more proportion (49.4\%) of the studied cases was living in the low household density area, compared with that of all the cases (45.3\%). Figure 2 shows the quartile distribution of four different mosquito indices. From this figure, difference was not observed among low, medium, and high household density areas.

Univariate analysis showed that $\mathrm{BI}, \mathrm{AI}, \mathrm{CI}$, and $\mathrm{HI}$ were significant (p-values < 0.05), with odds ratios of 1.02, 1.33, 1.04, and 1.01, respectively. Further stratified by the household density, BI was significant only in medium and high household density areas, with odds ratios of 1.03 and 1.05, respectively. Notably, both had a p-value $<0.001$. Similarly, HI was also significant (p-value $<0.05$ ) only in medium and high household density areas, with odds ratios of 1.03 and 1.04, respectively. AI was significant among all low, medium, and high household density areas, with odds ratios of 1.29, 1.45, and 1.3, respectively. CI was also significant among all low, medium, and high household density areas, with odds ratios of 1.02, 1.03 and 1.08. All AI and CI among all areas had a p-value $<0.001$ (Table 2).

Tables 3-5 show the estimates of odds ratio for each index (AI, BI, CI, or $\mathrm{HI}$ ), with each model adjusted by functions of rainfall, humidity, and temperature. Based on the results, $\mathrm{AI}, \mathrm{BI}, \mathrm{CI}$, and $\mathrm{HI}$ were significant, with odds ratios of $1.02,1.31,1.04$, and 1.02, respectively. In addition, all indices had a p-value $<0.001$. Further stratified by the household density, AI was significant among all low, medium, and high household density areas, with odds ratios of $1.29,1.49$, and 1.3 , respectively. All had a p-value $<0.0001$. BI was significant in medium and high household density areas, with odds ratios of 1.04 and 1.06, respectively. Notably, both had a p-value $<0.0001$. CI was also significant in medium and high household density areas, with odds ratios of 1.03 and 1.1 respectively. Both had a p-value $<0.0001$. HI was also significant in medium and high household density areas, with odds ratios of 1.07 and 1.04 , respectively. Both exhibited a p-value $<0.001$.

The effect of rainfall on the occurrence of dengue cases was classified into two. To consider the incubation period (3-8 days) after infection and the life cycle of mosquito, we regarded both the average rainfall of Week 2 before the diagnosis and that of Week 5 to Week 8 (5-8 week lag) before the diagnosis. A combination of a higher rainfall 5-8 weeks earlier and a lower rainfall 2 weeks earlier was related to higher probability of DF incidence. Meanwhile, higher relative humidity but lower temperature 2 weeks earlier was also related to higher DF incidence. Among all cases, $\mathrm{p}$-value $<0.001$ and the odds ratio between

\begin{tabular}{|c|c|c|c|c|c|c|}
\hline \multirow{2}{*}{$\begin{array}{c}\begin{array}{c}\text { Dengue } \\
\text { subjects } \\
\text { (Number) }\end{array} \\
\begin{array}{c}\text { Household } \\
\text { Density }^{\mathrm{a}}\end{array}\end{array}$} & \multicolumn{3}{|c|}{ Subjects recruited (2453) } & \multicolumn{3}{|c|}{ All subjects (4570) } \\
\hline & Low & medium & high & low & medium & high \\
\hline $\begin{array}{c}\text { number of DF } \\
(\%)\end{array}$ & 1212 & 565 & 676 & 2072 & 1082 & 1416 \\
\hline number of $\mathrm{Li}(\%)$ & 165 & 79 & 96 & 311 & 109 & 161 \\
\hline age $\left(\operatorname{std}^{b}\right)$ & $\begin{array}{c}46.6 \\
(19.5)\end{array}$ & $45.8(19.7)$ & $44.5(19)$ & $\begin{array}{c}45.8 \\
(19.2)\end{array}$ & $\begin{array}{c}44.6 \\
(19.6)\end{array}$ & $\begin{array}{c}44.2 \\
(18.3)\end{array}$ \\
\hline $\begin{array}{c}\text { gender ( } \% \text { of } \\
\text { male) }\end{array}$ & $\begin{array}{l}569: 643 \\
(1: 1.13)\end{array}$ & $\begin{array}{c}289: 276 \\
(1: 0.955)\end{array}$ & $\begin{array}{l}320: 356 \\
(1: 1.11)\end{array}$ & $\begin{array}{c}988: 1084 \\
(1: 1.1)\end{array}$ & $\begin{array}{c}542: 540 \\
(1: 1)\end{array}$ & $\begin{array}{l}650: 766 \\
(1: 1.18)\end{array}$ \\
\hline $\begin{array}{c}\text { population } \\
\text { density (pop/ } \\
\mathrm{km}^{2} \text { ) }\end{array}$ & 7998.36 & 12420.86 & 17408.46 & 5619.68 & 10484.72 & 17457.8 \\
\hline
\end{tabular}

alow household density: 1 household; medium household density: 2-10 households; high household density: $>10$ households.

${ }^{\mathrm{b}} \mathrm{std}$ : standard deviation

Table 1: Demographics of the studied cases by household density.
DF and the three levels of rainfall, temperature, and humidity either increased or decreased in a monotonic order (Tables 3-5).

\section{Discussion}

The difficulties in predicting dengue is complicated by the interplay among the vector, climate, social, economic, household environment, infected serotypes, and the individual susceptibility and immunity conditions. Therefore, an inconsistent results on the association between immature mosquito indices and DENV transmission has been repeatedly reported. Some study had found that higher BI, HI and CI are positively correlated with DF occurrence [31]; while others [32-35] found no significant or even negative [36,37] association. Given that mosquito density is temporally dynamic and spatially non-stationary [36], the following reasons may cause the inconsistency. (1) Lack of control of numerous environmental and demographic factors. (2) The household density may have interaction effect with BI on DF incidence. In our study, the case-crossover design was spatially and demographically matched, so all the factors not changed in short period were well-controlled. To resolve reason (2), we conducted separate analyses for different household density areas. The results showed that $\mathrm{BI}$ and $\mathrm{CI}$ had significant positive association with DF in medium and high household density (2 or more households per doorplate) areas, but not in the low household density areas. All other studies used pooled analysis which may dilute the effect and produced non-significant association.

The inference on the association between temperature and DF incidence is also inconsistent. A few studies $[37,38]$ also concluded that the temperature in a 3-month lag has negative association with the DF occurrence; and a study in Taiwan [39] also reported negative result. In contrast, Yu et al. [38] showed that minimum temperature in a 8-12-week lag is positively associated with DF cases. Shang et al. [27] demonstrated positive association in different lag periods. The inference on the association with rainfall is also inconsistent. Yu et al. [38] and Wu et al. [39] indicated that a 3-month lag rainfall is positively significant, whereas Chen et al. [37] described that the 3-month lag rainfall is negatively significant. Most of the results above reported negatively association between relative humidity and DF.

Our study integrated both meteorological and mosquito index data and suggested that both the short-lag (last 2 weeks, or 2-week lag) and long-lag (week 5-8 before, or 2-month lag) meteorological factors independently affect dengue case occurrence. Short-lag meteorological conditions of moderate rainfall, moderate temperature, and high humidity, in combination with a long-lag higher rainfall were related to higher probability of DF incidence. A possible explanation of the phenomenon is that the long-term heavier rainfall is responsible for creating a better environment for larvae to breed, and the short-lag moderate rainfall and temperature suitable for human's outdoor activities as well as for mosquito to feed. In contrast, short-lag heavy rainfall may be detrimental for mosquito.

\section{Limitations}

Due to Privacy Protection Regulation, the exact address cannot be accessed and the finest geographical information we can obtain is " $\mathrm{Li}$," which usually contains tens to hundreds of households. In addition, not all the Li's had frequent vector surveillance inspection. Some DF cases were forced to be excluded due to the incomplete vector surveillance in the residential Li within a month before the incidence. A potential selection bias was considered and missing at random was examined. No specific cluster or pattern was found with regard to the resident areas among the excluded cases. 
Citation: Tseng YT, Chang FS, Chao DY, Lian IB (2016) Re-Model the Relation of Vector Indices, Meteorological Factors and Dengue Fever. J Trop Dis 4: 200. doi:10.4172/2329-891X.1000200
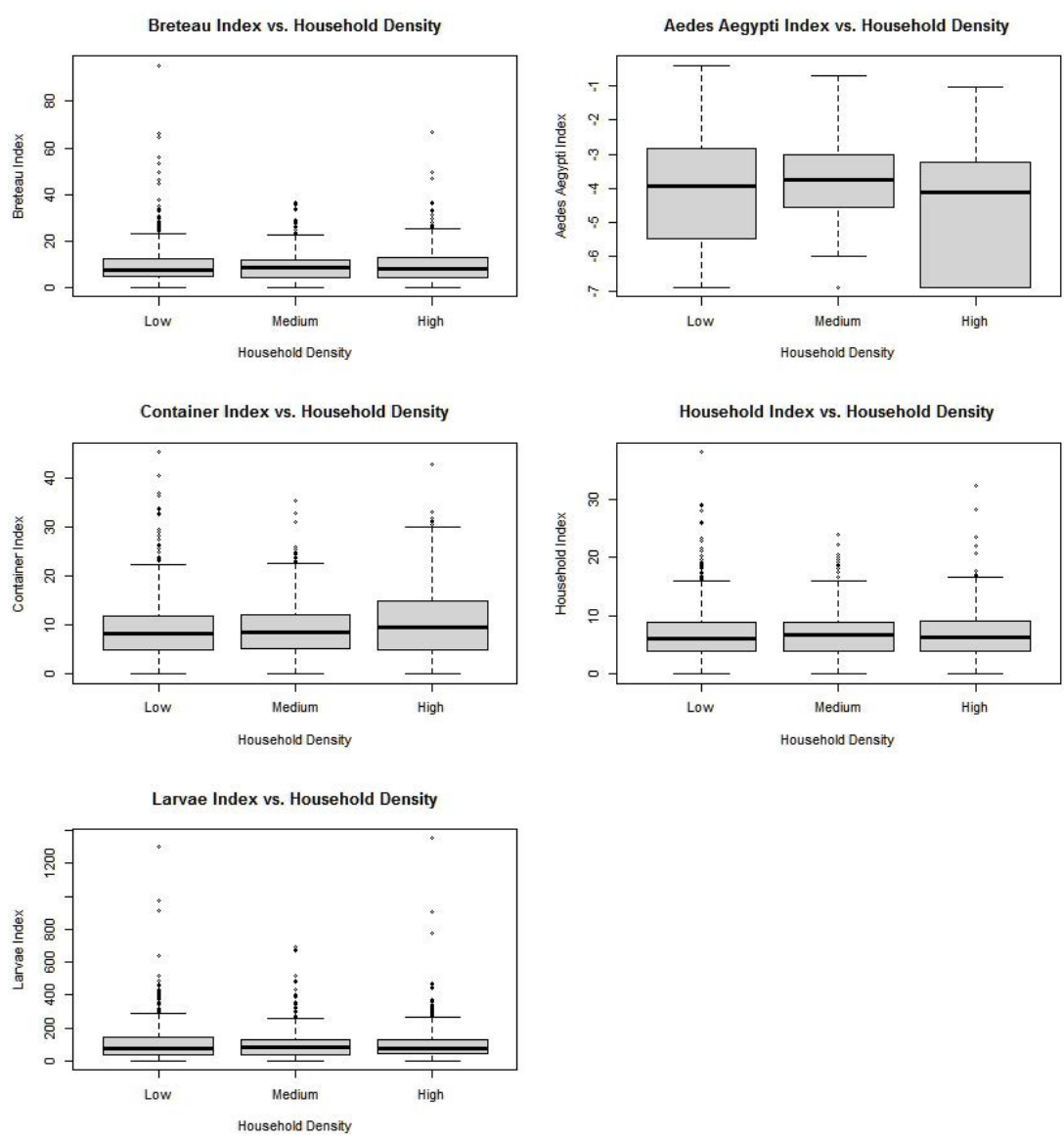

Figure 2: Box plots of various vector surveillance at low, medium, and high household density regions. Aedes aegypti index was log-transferred after adding 0.00 for avoiding infinity.

\begin{tabular}{|c|c|c|c|c|c|c|}
\hline & \multicolumn{2}{|c|}{ Low household density } & \multicolumn{2}{|c|}{ Medium household density } & \multicolumn{2}{|c|}{ High household density } \\
\hline & Odds ratio & $\mathbf{( 9 5 \%} \mathbf{~ C l})$ & Odds ratio & $\mathbf{( 9 5 \% ~ C l )}$ & Odds ratio & $(95 \%$ Cl) \\
\hline $\mathrm{BI}$ & 1.002 & $(0.994,1.01)$ & $1.03^{\mathrm{b}}$ & $(1.01,1.04)$ & $1.05^{\mathrm{a}}$ & $(1.03,1.06)$ \\
\hline $\mathrm{Al}$ & $1.29^{\mathrm{a}}$ & $(1.24,1.35)$ & $1.45^{\mathrm{a}}$ & $(1.36,1.55)$ & $1.32^{\mathrm{a}}$ & $(1.24,1.39)$ \\
\hline $\mathrm{Cl}$ & $1.02^{\mathrm{b}}$ & $(1.01,1.03)$ & $1.03^{\mathrm{b}}$ & $(1.01,1.04)$ & $1.08^{\mathrm{a}}$ & $(1.06,1.1)$ \\
\hline $\mathrm{HI}$ & 0.992 & $(0.977,1.01)$ & $1.03^{\mathrm{c}}$ & $(1.01,1.06)$ & $1.04^{\mathrm{b}}$ & $(1.02,1.06)$ \\
\hline
\end{tabular}

a p-value $<0.0001$

bp-value $<0.001$

${ }^{c} p$-value $<0.05$

Table 2: Result of univariate conditional logistic regression on vector by household density.

\begin{tabular}{|c|c|c|c|c|c|c|c|}
\hline & & \multicolumn{2}{|c|}{ Low household density ${ }^{a}$} & \multicolumn{2}{|c|}{ Medium household density ${ }^{a}$} & \multicolumn{2}{|c|}{ High household density ${ }^{a}$} \\
\hline & & Odds ratio & $(95 \% \mathrm{Cl})$ & Odds ratio & $(95 \% \mathrm{Cl})$ & Odds ratio & $(95 \% \mathrm{Cl})$ \\
\hline $\mathrm{BI}^{\mathrm{b}}$ & & 0.998 & $(0.988,1.01)$ & $1.04^{\mathrm{h}}$ & $(1.02,1.06)$ & $1.06^{9}$ & $(1.04,1.08)$ \\
\hline \multirow[b]{3}{*}{ 2-week lag rainfall ${ }^{c}$} & low & 1 & & 1 & & 1 & \\
\hline & medium & $0.247^{g}$ & $(0.198,0.307)$ & $0.584^{\mathrm{h}}$ & $(0.441,0.773)$ & $0.225^{9}$ & $(0.165,0.308)$ \\
\hline & high & $0.066^{9}$ & $(0.048,0.091)$ & $0.078^{\mathrm{g}}$ & $(0.052,0.119)$ & $0.04^{9}$ & $(0.025,0.064)$ \\
\hline
\end{tabular}


Citation: Tseng YT, Chang FS, Chao DY, Lian IB (2016) Re-Model the Relation of Vector Indices, Meteorological Factors and Dengue Fever. J Trop Dis 4: 200. doi:10.4172/2329-891X.1000200

Page 6 of 8

\begin{tabular}{|c|c|c|c|c|c|c|c|}
\hline \multirow{3}{*}{ 2-month lag rainfall ${ }^{\mathrm{d}}$} & low & 1 & & 1 & & 1 & \\
\hline & medium & $3.39^{9}$ & $(2.75,4.15)$ & $5.75^{\mathrm{g}}$ & $(4.04,8.17)$ & $3.68^{g}$ & $(2.7,5.02)$ \\
\hline & high & $7.42^{\mathrm{g}}$ & $(5.69,9.69)$ & $10.4^{\mathrm{g}}$ & $(6.96,15.4)$ & $5.04^{\mathrm{g}}$ & $(3.45,7.36)$ \\
\hline \multirow{3}{*}{ 2-week lag tempe } & low & 1 & & 1 & & 1 & \\
\hline & medium & $0.313^{g}$ & $(0.253,0.388)$ & $0.451^{\mathrm{g}}$ & $(0.334,0.61)$ & $0.181^{\mathrm{g}}$ & $(0.131,0.249)$ \\
\hline & high & $0.19^{g}$ & $(0.149,0.242)$ & $0.117^{g}$ & $(0.079,0.175)$ & $0.047^{g}$ & $(0.032,0.07)$ \\
\hline \multirow{3}{*}{ 2-week lag rh ${ }^{f}$} & low & 1 & & 1 & & 1 & \\
\hline & medium & $2.75^{\mathrm{g}}$ & $(2.2,3.44)$ & $1.78^{\mathrm{h}}$ & $(1.31,2.43)$ & $2.72^{\mathrm{g}}$ & $(2,3.69)$ \\
\hline & high & $6.82^{\mathrm{g}}$ & $(4.89,9.5)$ & $4.61^{\mathrm{g}}$ & $(3,7.07)$ & $5.09^{g}$ & $(3.32,7.8)$ \\
\hline
\end{tabular}

a low household density: 1 household; medium household density: 2-10 households; high household density: $>10$ households.

b BI: Breteau index

c 1-2-week lag rainfall: 8-14 day lag of accumulative rainfall. Low rainfall: 0-3.5 $\mathrm{mm}$, medium rainfall: $3.5-32 \mathrm{~mm}$, and high rainfall: $>32$.

d 5-8-week lag rainfall: $29-56$ day lag of accumulative rainfall. Low rainfall: 0-65 mm, medium rainfall: $65-240 \mathrm{~mm}$, and high rainfall: $>240 \mathrm{~mm}$.

e 1-2-week lag temp: 8-14 day lag of mean temperature (temp). Low temp: $18{ }^{\circ} \mathrm{C}-27.6{ }^{\circ} \mathrm{C}$, medium temp: $27.6{ }^{\circ} \mathrm{C}-29.1^{\circ} \mathrm{C}$, and high temp: $>29.1{ }^{\circ} \mathrm{C}$.

f 1-2-week lag rh: 8-14 day lag of mean relative humidity (rh). Low rh: 60\%-73.2\%, medium rh: 73.2\%-77.2\%, and high rh: $>77.2 \%$.

g p-value $<0.0001$.

h p-value $<0.001$

Table 3 : Results of multivariate conditional logistic regression on $\mathrm{BI}$ and meteorological factors by household density

\begin{tabular}{|c|c|c|c|c|c|c|c|}
\hline & & \multicolumn{2}{|c|}{ Low household density ${ }^{a}$} & \multicolumn{2}{|c|}{ Medium household density a } & \multicolumn{2}{|c|}{ High household density a } \\
\hline & & Odds ratio & $(95 \% \mathrm{Cl})$ & Odds ratio & $(95 \% \mathrm{Cl})$ & Odds ratio & $(95 \% \mathrm{Cl})$ \\
\hline $\mathrm{Al}^{\mathrm{b}}$ & & $1.29^{g}$ & $(1.23,1.36)$ & $1.49^{9}$ & $(1.37,1.61)$ & $1.3^{9}$ & $(1.21,1.39)$ \\
\hline \multirow{3}{*}{ 2-week lag rainfallc } & low & 1 & & 1 & & 1 & \\
\hline & medium & $0.224^{\mathrm{g}}$ & $(0.178,0.281)$ & $0.636^{\mathrm{h}}$ & $(0.474,0.854)$ & $0.236^{g}$ & $(0.173,0.323)$ \\
\hline & high & $0.06^{9}$ & $(0.043,0.084)$ & $0.092^{\mathrm{g}}$ & $(0.06,0.141)$ & $0.046^{g}$ & $(0.029,0.073)$ \\
\hline \multirow{3}{*}{ 2-month lag rainfall ${ }^{d}$} & low & 1 & & 1 & & 1 & \\
\hline & medium & $3.1^{\mathrm{g}}$ & $(2.5,3.85)$ & $5.84^{g}$ & $(4.03,8.47)$ & $3.89^{9}$ & $(2.85,5.3)$ \\
\hline & high & $6.99^{9}$ & $(5.31,9.2)$ & $10.6^{9}$ & $(7.01,16)$ & $4.9^{9}$ & $(3.36,7.15)$ \\
\hline \multirow{3}{*}{ 2-week lag tempe } & low & 1 & & 1 & & 1 & \\
\hline & medium & $0.304^{\mathrm{g}}$ & $(0.244,0.379)$ & $0.474^{g}$ & $(0.345,0.65)$ & $0.187^{g}$ & $(0.136,0.258)$ \\
\hline & high & $0.179^{g}$ & $(0.139,0.23)$ & $0.12^{\mathrm{g}}$ & $(0.08,0.18)$ & $0.052^{\mathrm{g}}$ & $(0.035,0.076)$ \\
\hline \multirow{3}{*}{ 2-week lag rh } & low & 1 & & 1 & & 1 & \\
\hline & medium & $2.54^{g}$ & $(2.02,3.18)$ & $1.83^{h}$ & $(1.32,2.54)$ & $3.11^{\mathrm{g}}$ & $(2.28,4.25)$ \\
\hline & high & $5.88^{g}$ & $(4.2,8.25)$ & $5.21^{g}$ & $(3.36,8.06)$ & $5.56^{g}$ & $(3.61,8.57)$ \\
\hline
\end{tabular}

a low household density: 1 household; medium household density: 2-10 households; high household density: >10 households.

${ }^{\mathrm{b}}$ Al: $\log$ Aedes aegypti index and plus positive small value.

c1-2-week lag rainfall: 8-14 day lag of accumulative rainfall. Low rainfall: 0-3.5 mm, medium rainfall: $3.5-32 \mathrm{~mm}$, and high rainfall: $>32$.

d 5-8-week lag rainfall: 29-56 day lag of accumulative rainfall. Low rainfall: 0-65 mm, medium rainfall: $65-240 \mathrm{~mm}$, and high rainfall: $>240 \mathrm{~mm}$.

e 1-2-week lag temp: 8-14 day lag of mean temperature (temp). Low temp: $18{ }^{\circ} \mathrm{C}-27.6{ }^{\circ} \mathrm{C}$, medium temp: $27.6^{\circ} \mathrm{C}-29.1^{\circ} \mathrm{C}$, and high temp: $>29.1^{\circ} \mathrm{C}$.

f 1-2-week lag rh: 8-14 day lag of mean relative humidity (rh). Low rh: $60 \%-73.2 \%$, medium rh: $73.2 \%-77.2 \%$, and high rh: $>77.2 \%$.

${ }^{9} \mathrm{p}$-value $<0.0001$.

h p-value $<0.001$

Table 4 : Results of multivariate conditional logistic regression on $\mathrm{BI}$ and meteorological factors by household density

\begin{tabular}{|c|c|c|c|c|c|c|c|}
\hline & & \multicolumn{2}{|c|}{ Low household density a } & \multicolumn{2}{|c|}{ Medium household density a } & \multicolumn{2}{|c|}{ High household density ${ }^{a}$} \\
\hline & & Odds ratio & $(95 \% \mathrm{Cl})$ & Odds ratio & $(95 \% \mathrm{Cl})$ & Odds ratio & $(95 \% \mathrm{Cl})$ \\
\hline $\mathrm{Cl}^{\mathrm{b}}$ & & 1.01 & $(0.995,1.02)$ & $1.03^{h}$ & $(1.01,1.06)$ & $1.1^{\mathrm{g}}$ & $(1.08,1.12)$ \\
\hline \multirow{3}{*}{ 2-week lag rainfall ${ }^{c}$} & low & 1 & & 1 & & 1 & \\
\hline & medium & $0.246^{g}$ & $(0.198,0.307)$ & $0.59^{\mathrm{h}}$ & $(0.446,0.78)$ & $0.202^{\mathrm{g}}$ & $(0.147,0.279)$ \\
\hline & high & $0.065^{9}$ & $(0.048,0.09)$ & $0.079^{9}$ & $(0.052,0.12)$ & $0.041^{g}$ & $(0.025,0.067)$ \\
\hline \multirow[b]{3}{*}{ 2-month lag rainfall ${ }^{d}$} & low & 1 & & 1 & & 1 & \\
\hline & medium & $3.35^{\mathrm{g}}$ & $(2.71,4.13)$ & $5.68^{g}$ & $(3.99,8.08)$ & $3.15^{\mathrm{g}}$ & $(2.3,4.31)$ \\
\hline & high & $7.23^{9}$ & $(5.53,9.44)$ & $10.3^{9}$ & $(6.93,15.4)$ & $4.5^{\mathrm{g}}$ & $(3.06,6.62)$ \\
\hline
\end{tabular}


Citation: Tseng YT, Chang FS, Chao DY, Lian IB (2016) Re-Model the Relation of Vector Indices, Meteorological Factors and Dengue Fever. J Trop Dis 4: 200. doi:10.4172/2329-891X.1000200

Page 7 of 8

\begin{tabular}{|c|c|c|c|c|c|c|c|}
\hline \multirow{3}{*}{ 2-week lag temp } & low & 1 & & 1 & & 1 & \\
\hline & medium & $0.316^{g}$ & $(0.255,0.391)$ & $0.454^{\mathrm{g}}$ & $(0.335,0.613)$ & $0.171^{\mathrm{g}}$ & $(0.123,0.237)$ \\
\hline & high & $0.19^{9}$ & $(0.15,0.242)$ & $0.123^{9}$ & $(0.083,0.182)$ & $0.048^{9}$ & $(0.032,0.072)$ \\
\hline \multirow{3}{*}{ 2-week lag rh } & low & 1 & & 1 & & 1 & \\
\hline & medium & $2.69^{g}$ & $(2.16,3.37)$ & $1.8^{\mathrm{h}}$ & $(1.3,2.46)$ & $2.72^{\mathrm{g}}$ & $(2,3.7)$ \\
\hline & high & $6.67^{\mathrm{g}}$ & $(4.79,9.28)$ & $4.91^{\mathrm{g}}$ & $(3.21,7.51)$ & $5.14^{\mathrm{g}}$ & $(3.33,7.94)$ \\
\hline
\end{tabular}

a low household density: 1 household; medium household density: 2-10 households; high household density: >10 households.

${ }^{\mathrm{b}} \mathrm{Cl}$ : Container index.

c 1-2-week lag rainfall: 8-14 day lag of accumulative rainfall. Low rainfall: 0-3.5 mm, medium rainfall: $3.5-32 \mathrm{~mm}$, and high rainfall: $>32$

d 5-8-week lag rainfall: 29-56 day lag of accumulative rainfall. Low rainfall: 0-65 mm, medium rainfall: $65-240 \mathrm{~mm}$, and high rainfall: $>240 \mathrm{~mm}$.

e 1-2-week lag temp: 8-14 day lag of mean temperature (temp). Low temp: $18{ }^{\circ} \mathrm{C}-27.6{ }^{\circ} \mathrm{C}$, medium temp: $27.6^{\circ} \mathrm{C}-29.1{ }^{\circ} \mathrm{C}$, and high temp: $>29.1{ }^{\circ} \mathrm{C}$.

f 1-2-week lag rh: 8-14 day lag of mean relative humidity (rh). Low rh: $60 \%-73.2 \%$, medium rh: $73.2 \%-77.2 \%$, and high rh: $>77.2 \%$.

g p-value $<0.0001$

${ }^{n}$ p-value $<0.001$

Table 5: Results of multivariate conditional logistic regression on $\mathrm{Cl}$ and meteorological factors by household density.

\section{Conclusion}

Meteorological conditions affect DF occurrence through the changes of mosquito density and biting behavior with a nonlinear relationship, and a single time-point rainfall variable in linear model may insufficiently fit. Our study suggested that short-lag conditions of moderate rainfall, moderate temperature and high humidity, in combination with a long-lag heavy rainfall were related to higher probability of DF incidence. BI and CI are useful predictors for DF occurrence in medium and high household density areas, but not in the low density (one households per doorplate) areas.

\section{Acknowledgments}

This work was supported by grants from Ministry of Science and Technology 103-2633-b-018-001, and the study was approved by Institutional Review Board IRB-R-05-002 from Taichung Hospital, Ministry of Health and Welfare. We thank the Department of Health, Kaohsiung City Government for offering data.

\section{References}

1. Nujum ZT, Thomas A, Vijayakumar K, Nair RR, Pillai MR, et al. (2014) Comparative performance of the probable case definitions of dengue by WHO (2009) and the WHO-SEAR expert group (2011). Pathog Glob Health 108: 103 110.

2. Simmons CP, Farrar JJ, van Vinh Chau N, Wills B (2012) Dengue. N Engl J Med 367:180-181.

3. Murray NE, Quam MB, Wilder-Smith A (2013) Epidemiology of dengue: past, present and future prospects. Clin Epidemiol 5: 299-309.

4. Liew C, Curtis CF (2004) Horizontal and vertical dispersal of dengue vector mosquitoes, Aedes aegypti and Aedes albopictus, in Singapore. Med Vet Entomol 18: 351-360.

5. Black WC 4th, Bennett KE, Gorrochótegui-Escalante N, Barillas-Mury CV, Fernández-Salas I, et al. (2002) Flavivirus susceptibility in Aedes aegypti. Arch Med Res 33: 379-388.

6. Watts DM, Burke DS, Harrison BA, Whitmire RE, Nisalak A (1987) Effect of temperature on the vector efficiency of Aedes aegypti for dengue 2 virus. Am J Trop Med Hyg 36: 143-152.

7. Breteau $H$ (1954) [Yellow fever in French West Africa; an aspect of masspreventive medicine]. Bull World Health Organ 11: 453-481.

8. Focks DA, Chadee DD (1997) Pupal survey: an epidemiologically significant surveillance method for Aedes aegypti: an example using data from Trinidad. Am J Trop Med Hyg 56: 159-167.

9. WHO Vector surveillance.

10. Guo S, Ling F, Hou J, Wang J, Fu G, et al. (2014) Mosquito Surveillance Revealed Lagged Effects of Mosquito Abundance on Mosquito-Borne Disease Transmission: A Retrospective Study in Zhejiang, China. PloS ONE 9: e112975.
11. Scott TW, Morrison AC (2010) Vector dynamics and transmission of dengue virus: implications for dengue surveillance and prevention strategies: vecto dynamics and dengue prevention. Curr Top Microbiol Immunol 338: 115-128.

12. Kay B, Vu SN (2005) New strategy against Aedes aegypti in Vietnam. Lance 365: 613-617.

13. Reiter P (2014) 25 Surveillance and Control of Urban Dengue Vectors. Dengue and dengue hemorrhagic fever.

14. Scott TW, Morrison AC (2003) Aedes aegypti density and the risk of dengue virus transmission. Ecological aspects for application of genetically modified mosquitoes 187-206.

15. Tun-Lin W, Kay BH, Barnes A (1995) Understanding productivity, a key to Aedes aegypti surveillance. Am J Trop Med Hyg 53: 595-601.

16. Tun-Lin W, Kay BH, Barnes A, Forsyth S (1996) Critical examination of Aedes aegypti indices: correlations with abundance. Am J Trop Med Hyg 54: 543-547.

17. Morrison A, Astete H, Chapilliquen F, Ramirez-Prada G, Diaz G, et al. (2004) Evaluation of a sampling methodology for rapid assessment of Aedes aegypt infestation levels in Iquitos, Peru. J Med Entomol 41: 502-510.

18. Scott TW, Morrison AC (2010a) Longitudinal field studies will guide a paradigm shift in dengue prevention. Vector Biology, Ecology and Control 139-161.

19. Bowman LR, Runge-Ranzinger S, McCall PJ (2014) Assessing the relationship between vector indices and dengue transmission: a systematic review of the evidence. PLoS Negl Trop Dis 8: e2848.

20. Chao DY, Lin TH, Hwang KP, Huang JH, Liu CC, et al. (2004) 1998 dengue hemorrhagic fever epidemic in Taiwan. Emerg Infect Dis 10: 552.

21. Chen HL, Lin SR, Liu HF, King CC, Hsieh SC, et al. (2008) Evolution of dengue virus type 2 during two consecutive outbreaks with an increase in severity in southern Taiwan in 2001-2002. Am J Trop Med Hyg 79: 495-505.

22. King CC, Wu YC, Chao DY, Lin TH, Chow L, et al. (2000) Major epidemics of dengue in Taiwan in 1981-2000: related to intensive virus activities in Asia. Dengue Bull 24: 1-10.

23. Shu PY, Su CL, Liao TL, Yang CF, Chang SF, et al. (2009) Molecula characterization of dengue viruses imported into Taiwan during 2003-2007: geographic distribution and genotype shift. Am J Trop Med Hyg 80: 1039-1046.

24. CDC (2009) Centers for Disease Control, R.O.C. Taiwan. Guidelines for dengue control.

25. Lin CH, Schiøler KL, Jepsen MR, Ho CK, Li SH, et al. (2012) Dengue outbreaks in high-income area, Kaohsiung City, Taiwan, 2003-2009. Emerg Infect Dis 18 : 1603-1611.

26. http://www.ey.gov.tw/en/

27. Shang CS, Fang CT, Liu CM, Wen TH, Tsai KH, et al. (2010) The role of imported cases and favorable meteorological conditions in the onset of dengue epidemics. PLoS Negl Trop Dis 4: e775.

28. CDC (2015) Centers for Disease Control, R.O.C. Taiwan. Case definition of dengue fever. 
Citation: Tseng YT, Chang FS, Chao DY, Lian IB (2016) Re-Model the Relation of Vector Indices, Meteorological Factors and Dengue Fever. J Trop Dis 4: 200. doi:10.4172/2329-891X.1000200

Page 8 of 8

29. Janes H, Sheppard L, Lumley T (2005) Case-crossover analyses of air pollution exposure data: referent selection strategies and their implications for bias. Epidemiology 16: 717-726.

30. Maclure M (1991) The case-crossover design: a method for studying transient effects on the risk of acute events. Am J Epidemiol 133: 144-153.

31. Pham HV, Doan HT, Phan TT, Minh NN (2011) Ecological factors associated with dengue fever in a Central Highlands province, Vietnam. BMC Infect Dis 11: 172.

32. Lowe R, Bailey TC, Stephenson DB, Jupp TE, Graham RJ, et al. (2013) The development of an early warning system for climate-sensitive disease risk with a focus on dengue epidemics in Southeast Brazil. Stat Med 32: 864-883.

33. Arboleda S, Jaramillo-O N, Peterson AT (2012) Spatial and temporal dynamics of Aedes aegypti larval sites in Bello, Colombia. J Vector Ecol 37: 37-48.

34. Sulaiman S, Pawanchee ZA, Arifin Z, Wahab A (1996) Relationship between Breteau and house indices and cases of dengue/dengue hemorrhagic fever in Kuala Lumpur, Malaysia. J Am Mosq Contrl Assoc Bull 12: 494-496.
35. Yew YW, Ye T, Ang LW, Ng LC, Yap G, et al. (2009) Seroepidemiology of dengue virus infection among adults in Singapore. Ann Acad Med Singapore 38: $667-675$.

36. Lin $\mathrm{CH}$, Wen TH (2011) Using geographically weighted regression (GWR) to explore spatial varying relationships of immature mosquitoes and human densities with the incidence of dengue. Int J Environ Res Public Health 8: 2798 2815.

37. Chen SC, Liao CM, Chio CP, Chou HH, You SH, et al. (2010) Lagged temperature effect with mosquito transmission potential explains dengue variability in southern Taiwan: insights from a statistical analysis. Sci Tota Environ 408: 4069-4075.

38. Yu HL, Yang SJ, Yen HJ, Christakos G (2011) A spatio-temporal climate-based model of early dengue fever warning in southern Taiwan. Stoch Environ Res Risk Assess 25: 485-494.

39. Wu PC, Guo HR, Lung SC, Lin CY, Su HJ (2007) Weather as an effective predictor for occurrence of dengue fever in Taiwan. Acta Trop 103: 50-57. 